\title{
Habituation and recovery of orienting in rats as a function of stimulus significance
}

\author{
JOHN G. M. EVANS \\ Monash University, Clayton, Victoria, Australia \\ and \\ GEOFFREY R. HAMMOND \\ University of Western Australia, Nedlands, Western Australia, Australia
}

\begin{abstract}
Three experiments measured orienting of rats to stimuli varying in intrinsic significance. In the first, rats showed greater reactivity to a recording of a conspecific's distress squeal than to a simulated mimic squeal when the stimuli were presented at $100 \mathrm{~dB}$ but not at $80 \mathrm{~dB}$, and orienting to all stimuli habituated rapidly with repeated stimulus exposures. Experiment 2 showed that, following several stimulus exposures, recovery of orienting after rest periods of 1 and 7 days was a function of the stimulus: Orienting to the distress squeal presented at $100 \mathrm{~dB}$ recovered more rapidly than did orienting to the mimic squeal at $100 \mathrm{~dB}$ or to either the mimic or the distress squeal presented at $80 \mathrm{~dB}$. Experiment 3 showed that habituation to the most significant stimulus, the distress squeal presented at $100 \mathrm{~dB}$, was retarded at long (24-h) interstimulus intervals, presumably a consequence of less stimulus-to-stimulus transfer of habituation with this stimulus. The results are discussed in terms of biological constraints on habituation of orienting and on recovery of orienting following habituation.
\end{abstract}

Much recent work on animal learning has been influenced by the ways in which the hereditary propensities of a particular species might influence the rate at which particular tasks are acquired or, indeed, whether they will be acquired at all. The ideas and relevant experimental evidence have been widely reviewed (e.g., Bolles, 1970; Dunham, 1977; Hinde \& Stevenson-Hinde, 1973; Rozin \& Kalat, 1971; Seligman, 1970; Shettleworth, 1972). This work supports the general statement by Hebb (1972) that "heredity makes some kinds of learning easy or inevitable, others hard, and thus guides learning" (p. 127). A central idea in this approach is that behavior that can be seen as adaptive is acquired readily, whereas behavior that is maladaptive is acquired slowly, if at all.

In this view, individually acquired behavior is part of an organism's adaptation to its environment, and as such is acquired at a rate that is influenced strongly by the adaptive value of the behavior. It is likely that retention of learning, as well as initial acquisition, is influenced by the adaptiveness of the learning (Rozin \& Kalat, 1971). That is, if some behavior is adaptive, it may be acquired quickly and be retained for a relatively long time; a behavior that does not contribute to adaptation, or is maladaptive,

Send reprint requests to Geoffrey R. Hammond, Department of Psychology, University of Western Australia, Nedlands, Western Australia 6009, Australia. may be acquired slowly and be retained for less time. The operation of biological propensities on both acquisition and retention would give, in this way, a surer adaptation of an organism's acquired behavior to environmental demands.

Evidence that retention is influenced in a way similar to acquisition comes from Figler (1972), who studied acquisition and retention over $24 \mathrm{~h}$ of habituation of components of the threat display of Siamese fighting fish to a variety of elicitors. He reported that retention of habituation was an inverse function of the initial potency of the elicitor: Habituation following sustained exposure to an initially strong elicitor (a live, reactive male) recovered over the 24 -h rest interval, whereas habituation to a relatively weak elicitor (such as a cut-out model) did not recover. Figler's results suggest that habituation to a releasing stimulus is short-lived, whereas habituation to approximations of the releasing stimulus is retained longer.

A number of experiments reviewed by Hinde (1970) show a rapid (although sometimes incomplete) recovery of responsiveness following habituation to repeated presentations of a variety of releasing stimuli. In contrast, Zeiner and Peeke (1969), using a licksuppression method, did not find evidence of recovery of orienting in rats after retention intervals of 3 and 7 days following habituation to conspecific distress signals. Their failure to find recovery may have resulted from the relatively large number of training trials or perhaps from the severity of the deprivation schedule used. As they noted, duration 
of suppression was an inverse function of deprivation level; severe deprivation levels may therefore have acted to minimize orienting.

The present three experiments used an adaptation of Zeiner and Peeke's (1969) lick-suppression technique as a measure of orienting to auditory signals. Experiment 1 repeated and extended Peeke and Zeiner's (1970) findings of differential habituation to stimuli that differed in biological importance. Experiments 2 and 3 gave evidence of differential retention of habituation to stimuli that differed in biological importance.

\section{EXPERIMENT 1}

Peeke and Zeiner (1970) found more pronounced suppression of licking in rats following presentation of conspecific distress calls than following pure tones at the dominant frequency of the distress calls $(1.5 \mathrm{kHz})$. As they pointed out, this difference could have resulted from the "informational difference" between the two stimuli, or perhaps from the difference in stimulus complexity. Previous work in our laboratory had compared habituation to distress squeals with habituation to mimic squeals made as a closer acoustic simulation of the distress squeals. This showed that differential reactivity between stimuli was usually maintained under these conditions. Two results were of interest. First, observation of the animals' behavior during a habituation session showed that duration of lick suppression following stimulus presentation was not correlated highly with orienting behavior. Frequently, animals would stop licking immediately after onset of the stimulus and would orient toward the source of the stimulus or actively search around the small experimental chamber. Following this period of orienting or searching, animals would not necessarily return to drinking, but of ten would engage in other behaviors, chiefly grooming. In this way, measures of lick suppression gave a variable overestimate of stimulus-linked attentive behavior. To remove this source of variability, we videotaped behavior and scored the duration of stimulus-linked attentive behavior from these records. Second, although reactivity to distress squeals was always greater than that to the mimic squeals, the difference was not always statistically reliable. This may have resulted from using a stimulus of a lower intensity (from about 82-87 dB re .0002 dynes/ $\left.\mathrm{cm}^{2}\right)$ than that used by Peeke and Zeiner $(100 \mathrm{~dB}$, with the same reference). Using the more direct measure of orienting, Experiment 1 compared habituation to distress and mimic squeals presented at two intensities, 80 and $100 \mathrm{~dB}$, both re .0002 dynes $/ \mathrm{cm}^{2}$.

\section{Method}

Subjects. Thirty-two male albino rats that weighed $271-455 \mathrm{~g}$ (mean $=333 \mathrm{~g}$ ) and that were $168-172$ days old at the time of

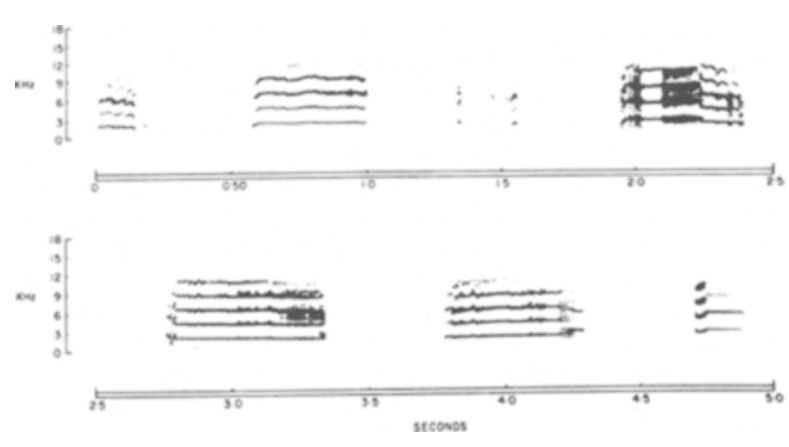

Figure 1. Sonagram of the distress squeal.

habituation were housed two per cage. A 12-h:12-h light:dark cycle was imposed, and the temperature was restricted to $21^{\circ} \mathrm{C}$ $23^{\circ} \mathrm{C}$. Food was continually available throughout the study, and water was restricted as described below.

Apparatus. The test and holding cages were made of white polyvinyl chloride. The test cages were rectangular $(15 \times 32 \mathrm{~cm})$, and the holding cages were square $(30 \times 30 \mathrm{~cm})$. All were $12 \mathrm{~cm}$ deep, had mesh tops, and had sawdust on the floor. At one end of each test cage was a stainless steel ballspout attached to a water bottle. This spout passed through an electrical coil, and as the rat licked, a magnet attached to the ball was deflected, which created a small voltage that was amplified and recorded by a Grass polygraph (Model 7B). A television camera was mounted above the test cages and connected to a videotape recorder to obtain continuous records of the animals' licking and other behaviors.

The stimuli used were recordings of a rat squeal and a mimic squeal. The rat squeal was taped from an animal being given an intraperitoneal injection. A sonagram (Kay Sona-Graph 6061D) of the squeal determined its duration and component frequencies. As can be seen in Figure 1, total duration of the squeal was $5.0 \mathrm{sec}$. It was broken into seven bursts of noise that varied in duration from 150 msec (Bursts 1 and 3) to $600 \mathrm{msec}$ (Burst 5), with interburst intervals of about $400 \mathrm{msec}$ (range $=380$ to $460 \mathrm{msec}$ ). The major frequencies were around $2325,4347,6666,9000$, and $11,250 \mathrm{~Hz}$. A mimic squeal was constructed by simultaneously recording square waves (to obtain the harmonics apparent in the sonagram) of these frequencies. They were generated by Exact function generators (Model 10383) and were spliced into a tape to simulate the rat squeal. The temporal structure of each squeal is given in Table 1. In some cases in which there was minor intensity variation between corresponding bursts of the distress and mimic squeals, the mimic squeal was always more intense. Any resultant bias was therefore toward greater reactivity to the mimic squeal, which gave a conservative test of the hypotheses.

The amplified stimuli were replayed to the animals during the habituation session through a speaker $10 \mathrm{~cm}$ in front of and $50 \mathrm{~cm}$

Table 1

Duration (in Milliseconds) of the Bursts of Noise and the Interburst Intervals (IBI, in Milliseconds) for the Distress and Mimic Squeals

\begin{tabular}{cccccc}
\hline \multirow{2}{*}{$\begin{array}{c}\text { Burst/IBI } \\
\text { Number }\end{array}$} & \multicolumn{2}{c}{ Distress Squeal } & & \multicolumn{2}{c}{ Mimic Squeal } \\
\cline { 2 - 3 } \cline { 5 - 6 } & Burst & IBI & & Burst & IBI \\
\hline 1 & 150 & 440 & & 150 & 440 \\
2 & 440 & 420 & 440 & 410 \\
3 & 150 & 390 & 150 & 390 \\
4 & 460 & 380 & 460 & 380 \\
5 & 600 & 460 & 600 & 460 \\
6 & 500 & 430 & 500 & 440 \\
7 & 180 & & 160 & \\
\hline
\end{tabular}


above the drinking spout. A voice relay was on line to detect the stimulus as it was played. This activated the signal recorder on the polygraph, thus ensuring that the temporal position of the stimulus was recorded in relation to the animals' licking. For the same reason, the videotape recorder was also on line.

Procedure. Habituation was measured with the lick-suppression technique. The rats were trained to lick at a spout, and, while they were licking, the stimulus to be habituated to was presented. The total lick suppression due to orienting to the stimulus was measured on each trial by modifying the lick suppression recorded on the polygraph by the videotape recording. Nonlicking not due to orienting was identified and removed from polygraph recording.

Three categories of orienting were defined: (1) stationary orienting - the animal stopped drinking and looked up toward the speaker; (2) mobile orienting - the animal stopped drinking, looked up toward the speaker, and moved around the cage, constantly reorienting toward the speaker; (3) freezing-the animal initially oriented toward the speaker but then remained rigid before either returning to drink or engaging in some other activity. Thus, both orienting durations and behavioral category judgments were obtained. All category judgments were made by a single observer.

The rats were randomly assigned to one of four groups ( $n=8$ per group). Each group was given 10 exposures to either the distress (D) or the mimic (M) squeals at either 80 or $100 \mathrm{~dB}$, as measured at the spout. The groups were designated D80, M80, D100, and M100.

To overcome the novelty of the situation and to ensure that the animals would drink during each habituation trial, the rats received 5 days of drink training in the test apparatus prior to habituation. Each animal was given two drinks per day, each of 30-min duration, at $5 \mathrm{~h}$ into the light cycle and at $1 \mathrm{~h}$ into the dark cycle $(5 \mathrm{~h} \mathrm{~L}$ and $1 \mathrm{~h}$ D). The drink at $5 \mathrm{~h} \mathrm{~L}$ was in the test chamber, and the drink at $1 \mathrm{~h}$ $D$ was in the home cage. The animals received a $0.009 \mathrm{M}$ saccharin solution in the test chamber (to ensure continuous drinking) and water in their home cages. This deprivation schedule resulted in a $2.5 \%$ to $8.6 \%$ weight loss by the time of the habituation session.

On the day the habituation session was run, the scheduled drinks were omitted, and the animals were allowed access to the saccharin solution only during the habituation trials. On each trial, the animal was placed in the test cage, and after it had licked continuously for about $10 \mathrm{sec}$, the recording equipment was switched on and the appropriate stimulus was presented. After the animal had resumed drinking following the stimulus-induced disruption, the equipment was turned off and the rat was removed from the test cage and placed in the holding cage with its home-cage mate for the duration of the intertrial interval. Cage mates were run simultaneously in separate chambers. Three minutes later, each animal was removed from the holding cage, and was replaced in the test apparatus, and the above procedure was repeated. Ten trials were given, following which the rats were returned to their home cages. The above procedure resulted in a variable-interval interstimulus interval (ISI) (offset to onset) for which the mean was $3 \mathrm{~min} 43 \mathrm{sec}$ and the range was from $3 \mathrm{~min} 25 \mathrm{sec}$ to $4 \mathrm{~min}$ $21 \mathrm{sec}$. Habituation sessions were from $10 \mathrm{~h} \mathrm{~L}$ to $1 \mathrm{~h} \mathrm{D}$. Testing time was counterbalanced across the groups.

\section{Results and Discussion}

Figure 2 shows the median orienting duration (in seconds) on each trial for the stimulus groups. Since there were no differences between the groups tested in the light and dark cycles, the data for these conditions were combined. Habituation of orienting was evident in each group (for trials, $\chi_{\mathrm{r}}^{2}=38.19, \mathrm{p}<.05$ ). ${ }^{1}$ Both stimulus intensity ( $80 \mathrm{vs.} 100 \mathrm{~dB})$ and stimulus quality ( $M$ vs. $D$ ) influenced orienting duration taken over all trials, with greater reactivity to the louder

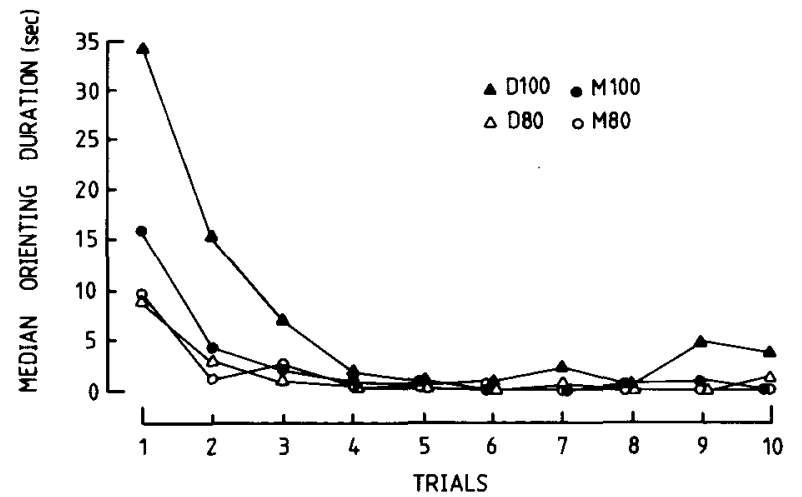

Figure 2. Median orienting duration (in seconds) for the four stimulus groups across the 10 stimulus exposures in Experiment 1.

stimulus (median orienting duration $=.10$ and $1.84 \mathrm{sec}$ for the 80- and 100-dB stimuli, respectively, $\mathrm{U}=3, \mathrm{p}<.05$ ) and to the distress call (median orienting duration over all trials $=.76$ and $1.46 \mathrm{sec}$ for the mimic and distress squeals, respectively, $\mathrm{U}=0, \mathrm{p}<.05$ ). These differences were due largely to the heightened activity of the D100 group compared with the reactivity of the other three groups. That is, the effect of stimulus quality was intensity dependent; more prolonged orienting to the distress than to the mimic squeal was found only at the higher of the two intensities (stimulus quality $x$ stimulus intensity interaction, $U=8, p<.05$ ). Although some animals exposed to the 80-dB stimuli continued to orient to the stimuli for brief periods, median duration of orienting for these two stimulus groups over Trials 8-10 was zero. Slightly greater terminal levels of orienting were evident to the 100 $\mathrm{dB}$ stimuli (group median orienting durations $=.32$ and $3.10 \mathrm{sec}$ for the $M$ and $D$ squeals, respectively).

Response-category data from the initial stimulus presentation were consistent with the orienting durations recorded on that trial. More animals froze to the D100 stimulus than to the M100 stimulus (7 and 1 , respectively, $p=.01$, Fisher exact test). The remaining animals in these groups and all animals in the $80-\mathrm{dB}$ stimulus groups either mobile-oriented or stationary-oriented. Over subsequent trials, all animals responded similarly to the various stimuli, and there were no reliable statistical differences between the distributions of any of the groups.

These results add to those of Peeke and Zeiner (1970). First, they confirm Peeke and Zeiner's finding that a distress call can provoke greater reactivity than can a simulation of the distress call, and that this reactivity habituates quickly with repetition of the stimulus; second, they extend Peeke and Zeiner's finding by showing that the increased reactivity to a distress call is intensity limited; and third, in that the mimic call was a closer simulation of the distress call 
than was the pure tone used by Peeke and Zeiner, these results suggest that the greater orienting provoked by the distress call is a result of stimulus meaning rather than of simple acoustic complexity.

\section{EXPERIMENT 2}

Experiment 2 was designed to test whether habituation to the various stimuli used in Experiment 1 would be retained differentially according to the biological significance of the habituated stimulus. It was expected that habituation to biologically important stimuli, which could be behaviorally maladaptive, would be retained less strongly than habituation to an acoustically similar, but biologically neutral, stimulus. In order to make unconfounded comparisons of retention performance between groups, it is necessary to establish equivalent acquisition between the groups at the end of training. Because reactivity was found to be greater to the D100 stimulus than to the other stimuli, different amounts of habituation training were given in order to reach equivalent levels of reactivity. Based upon the results of Experiment 1, the animals exposed to the D100 stimulus were given three habituation trials, and the remainder (M100, $\mathrm{D} 80$, and $\mathrm{M80}$ ) were given two habituation trials. Following this treatment, retention tests using the habituated stimulus were given immediately or at intervals of 1 or 7 days.

\section{Method}

Subjects. The subjects were 120 male albino rats that weighed between 194-332 $\mathrm{g}$ (mean $=263 \mathrm{~g}$ ) and that were $105-110$ days old at the time of the retention test. Housing conditions were those described for the previous experiment.

Apparatus. The apparatus was that used in Experiment 1.

Procedure. The method and measures of habituation were those used in the previous experiment. Each animal was habituated and, after its designated retention interval, given three test (rehabituation) trials.

The subjects were assigned randomly to one of 12 groups $(n=$ 10 per group) defined by stimulus quality (M or D), stimulus intensity (80 or $100 \mathrm{~dB})$, and retention interval (RI) $(0,1$, or 7 days). Thus, the 80D7 group, for example, was habituated to the D stimulus at $80 \mathrm{~dB}$ and was given a retention test with the same stimulus at the same intensity 7 days later.

The animals were given 3 days of drink training (as described previously) in the test apparatus prior to habituation. The scheduled drinks were omitted during the experimental sessions, and the animals had access to water only during the test trials. In the habituation session, the animals in the 80-dB and the M100 groups received two habituation trials, whereas those in the D100 groups received three. This procedure was used to give equal reactivity on the next trial if habituation were continued. The 0 RI groups received three test trials immediately (as though it were continuous acquisition). The remaining animals (the 1- and 7-day RI groups) were returned to their home cages for the duration of their respective RIs. The 1-day RI groups were given access to water for $1 \mathrm{~h}$, and the 7-day RI groups were given continuous access to water upon being returned to their home cages. The 7-day RI groups were deprived of water $24 \mathrm{~h}$ before their retention test. This deprivation schedule resulted in $3.4 \%$ to $13.9 \%$ weight loss by the time of the retention test.
The procedure followed for the three test trials was the same as that for the habituation trials. Both the habituation and the retention-test sessions were conducted between 9 and $12 \mathrm{~h} \mathrm{~L}$, with testing time counterbalanced across groups. The mean ISI was $3 \mathrm{~min} 3 \mathrm{sec}$, and the mean RI for the 0 group was $3 \mathrm{~min} 41 \mathrm{sec}$.

\section{Results and Discussion}

Figure 3 shows the median orienting duration for each stimulus group over five trials for the immediateretention groups. These trials are made up of three acquisition trials and two retention trials for the D100 stimulus group and two acquisition trials and three retention trials for the D80, M80, and $\mathrm{M} 100$ stimulus groups. Because the RI for these four groups was the same as the ISI in the habituation and retention test sessions, these data can be treated as coming from five acquisition trials. The data from the third retention trial for the D100 group (its sixth "acquisition trial") have been omitted for simplicity of presentation. Treated as acquisition data, these results replicate those of Figure 2 in terms of both absolute level of orienting and rate of habituation of orienting across trials. As in Experiment 1, there were statistically reliable effects of trials $\left[\chi_{\mathrm{r}}^{2}(4)=\right.$ 13.40, $\mathrm{p}<.05)$ and stimulus intensity $(U=2, p<$ $.05)$. The effect of stimulus quality did not reach significance $(U=27, p=.10)$.

There was only minor variation among the median scores of these four stimulus groups on their first retention trial the (fourth "acquisition trial" for the D100 group and the third "acquisition trial" for the remaining three groups). These median scores ranged from $1.25 \mathrm{sec}$ for the D80 group to $3.24 \mathrm{sec}$ for the M100 group. Although it cannot be determined for the 1- and 7-day retention groups, it seems likely from these data that the additional acquisition trial given to the D100 group was sufficient to establish equal reactivity across groups before imposition of the RI.

Median orienting duration on the first retention trial for each of the four stimulus groups at each of

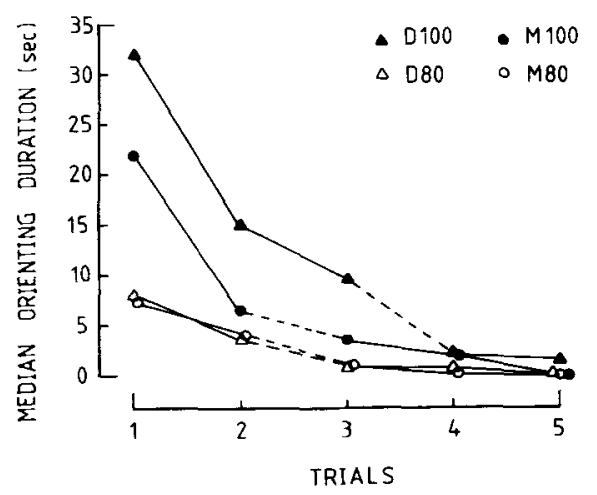

Figure 3. Median orienting duration (in seconds) for the four stimulus groups tested at the 0-day retention interval across five stimulus exposures in Experiment 2. 
the three RIs is shown in Figure 4. Overall, there was progressive recovery of orienting across the RIs. This is supported by a reliable main effect of RI for both intensity groups $[H(2)=17.00$ and $H(2)=12.19$, ps $<.05$, for the 80- and 100-dB stimulus groups, respectively]. Thus, the effects of habituation dissipated progressively across the RIs, as if the subjects were forgetting their previous exposure to the stimuli.

More importantly, there was differential recovery of orienting to the distress and mimic squeals within the 100-dB intensity level: Orienting recovered to a higher level in the D100 group than in the M100 group, although both groups showed equivalent amounts of orienting when tested immediately following the habituation trials [stimulus quality $\times$ RI interaction, $\mathrm{H}(2)=6.98, \mathrm{p}<.05$ ). Although duration of orienting to the $80-\mathrm{dB}$ distress and mimic squeals diverged across RIs in the expected direction, the interaction failed to reach statistical significance $[\mathrm{H}(2)$ $=1.24, \mathrm{p}>.05]$.

Figure 3 and Figure 4 together indicate that even following the 7-day RI, the orienting duration of the D100 group had not returned to the level shown on the first habituation trial. This can be attributed largely to changes in the form of the stimulus-linked behavior as a function of stimulus exposure. On the first presentation of the D100 stimulus, 24 of the 30 animals froze; on the first retention trial, only 5 of the 30 animals receiving the D100 stimulus froze, and of these, one belonged to the 7-day RI group. Thus, although orienting recovered markedly in the animals receiving the D100 stimulus, it recovered to a less extreme form of defensive behavior, and in only relatively few instances did freezing reappear. For this reason, it seems invalid to portray a subject's orienting duration on retention trials as a proportion of its orienting duration on the first stimulus presentation, as such a comparison ignores the often long times associated with freezing, a behavior seen predominantly following the first stimulus exposure.

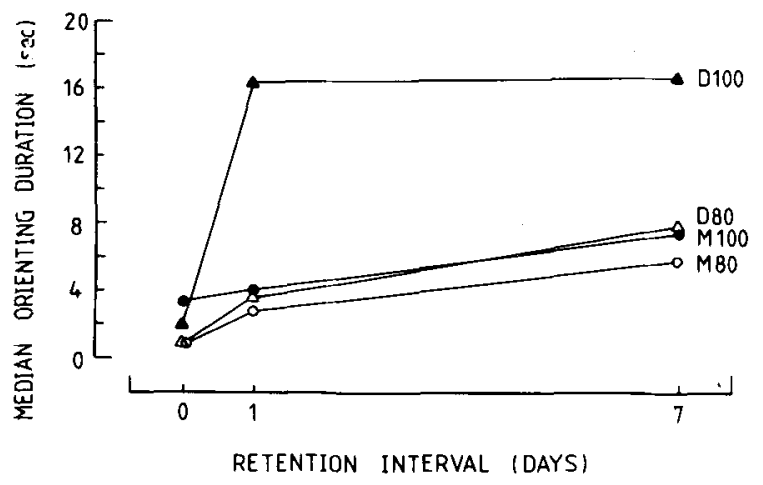

Figure 4. Median orienting duration (in seconds) for the four stimulus groups on the first stimulus exposure following the retenton interval in Experiment 2.
Data from the three retention trials are presented in full, since they show the same pattern as data from the first retention trial alone. As expected, no recovery was evident for any stimulus group at the immediate RI; at the 1-day RI, only the D100 group showed recovery, and this was confined to the first retention trial; at the 7-day RI, the M80, D80, and M100 groups showed recovery on the first trial, and the D100 group showed recovery on the first two retention trials before rehabituation was complete. No statistical conclusion was changed by including all three trials in the analysis.

\section{EXPERIMENT 3}

If the result of Experiment 2 (differential recovery in orienting over $24 \mathrm{~h}$ according to biological significance of the stimulus) has generality, then in contrast with development of habituation to a biologically unimportant stimulus, development of habituation to a biologically important stimulus should be sensitive to variations in ISI between several minutes and $24 \mathrm{~h}$. There should be less transfer of habituation across trials for repeated presentations of a biologically important than for an unimportant stimulus, and hence slower development of habituation across trials. In this experiment, habituation of orienting to daily exposures to the four stimuli used previously was measured.

\section{Method}

Subjects. Thirty-two male albino rats that weighed 219-326 g (mean $=280 \mathrm{~g}$ ) and that were $105-107$ days old at the start of the experiment were housed under the conditions described in Experiment 1.

Apparatus and Procedure. The method and measures of habituation, and the apparatus, were those described in the first experiment.

The animals were assigned randomly to one of four groups ( $n=8$ per group) defined by stimulus quality ( $D$ or $M$ squeals) and intensity (80 or $100 \mathrm{~dB}$ ). The groups were designated D80, M80, $\mathrm{D} 100$, and M100. All underwent 3 days of drink training as described in Experiment 1, with the exception that the saccharin solution was replaced with water. On the 4th day, the first habituation trial was given in place of the $5 \mathrm{~h} \mathrm{~L}$ drink. After the trial, the animals were returned to their home cages and given access to water for $30 \mathrm{~min}$. The drink at $1 \mathrm{~h} \mathrm{D}$ remained unchanged. Each animal received 10 such trials at $24 \mathrm{~h}$ intervals. By the end of the experiment, the amount of weight gained by the rats varied from $9 \%$ to $23 \%$ (mean $=14 \%)$.

All trials were conducted between 5 and $6 \mathrm{~h} \mathrm{~L}$ for the 80-dB groups and between 6 and $7 \mathrm{~h} \mathrm{~L}$ for the 100-dB groups. Within each intensity level, the time of testing individuals was counterbalanced across groups.

\section{Results and Discussion}

Figure 5 shows that habituation of orienting to the four stimuli presented with a 24-h ISI was similar to that observed in Experiments 1 and 2. There was, however, one important exception: Greater reactivity to the D100 stimulus compared with that to the M100 stimulus was evident on Trials 1-6 inclusive (all Us < 


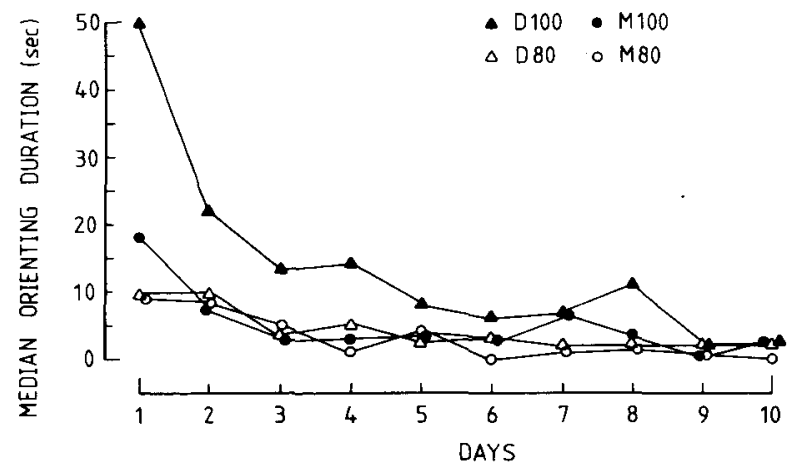

Figure 5. Median orienting duration (in seconds) for the four stimulus groups across the $\mathbf{1 0}$ daily stimulus exposures in Experiment 3 .

15.5, ps $<.05)$, whereas the D100 stimulus elicited greater reactivity than did the M100 stimulus on only the first trial in Experiment 1 and on the first three trials in Experiment 2. The more sustained differential orienting to the D100 and M100 stimuli in Experiment 3 was not a result of increased initial reactivity to the D100 stimulus. Orienting to the D100 stimulus on the first trial in Experiment 3 was not reliably greater than orienting to the same stimulus on the first trial in either Experiment 1 or Experiment $2(U=20$ and 27 , respectively, both ps $>.10)$. Median orienting durations (in seconds) to the M100 and D100 stimuli on Trials 4 and 5 across the three experiments are given in Table 2. Trials 4 and 5 are shown because only five trials were given in Experiment 2. Unlike orienting to the M100 stimulus, which was not much affected by a change in ISI from several minutes to $24 \mathrm{~h}$, orienting to the D100 stimulus was increased in Experiment 3 over that in Experiments 1 and 2 . It is evident from these crossexperiment comparisons that potentiation of orienting to the D100 stimulus presented with a 24-h ISI was relatively weak and transitory. There is some support, however, for the expectation that development of habituation to the D100 stimulus would be slower with stimulus presentations spaced $24 \mathrm{~h}$ apart. The insensitivity of the biologically unimportant

Table 2

Group Median Orienting Duration (in Seconds) to the M100 and D100 Stimuli on Trials 4 and 5 Across the Three Experiments

\begin{tabular}{|c|c|c|c|}
\hline \multirow[b]{2}{*}{ Stimulus } & \multicolumn{3}{|c|}{ Experiment } \\
\hline & 1 & 2 & 3 \\
\hline \multicolumn{4}{|c|}{ Trial 4} \\
\hline $\begin{array}{l}\text { M100 } \\
\text { D100 }\end{array}$ & $\begin{array}{r}.72 \\
2.00\end{array}$ & $\begin{array}{l}1.88 \\
2.00\end{array}$ & $\begin{array}{r}2.82 \\
14.23\end{array}$ \\
\hline \multicolumn{4}{|c|}{ Trial 5} \\
\hline $\begin{array}{l}\text { M100 } \\
\text { D100 }\end{array}$ & $\begin{array}{l}.32 \\
.84\end{array}$ & $\begin{array}{c}0 \\
1.58\end{array}$ & $\begin{array}{l}3.60 \\
8.12\end{array}$ \\
\hline
\end{tabular}

stimuli used here to the wide variation in ISI is consistent with another report in the literature: File (1973) showed that habituation of orienting to a 77.5dB pure tone increased with increasing ISI from .25 to $2 \mathrm{~min}$ and that further increases in ISI from $2 \mathrm{~min}$ to $24 \mathrm{~h}$ were without effect. Orienting over the last three stimulus exposures was somewhat more prolonged than that recorded in Experiment 1, with the D100 stimulus again evoking greatest orienting (group medians, in seconds: $\mathrm{M} 80=.54 ; \mathrm{D} 80=2.24$; $\mathrm{M} 100=1.68 ; \mathrm{D} 100=5.50$ ).

\section{GENERAL DISCUSSION}

The present results illustrate the importance of the nature of the stimulus in habituation: Provided the stimuli are sufficiently intense, distress squeals provoke greater reactivity than do quite close acoustic simulations, but in both cases reactivity habituates within a few trials. Rapid habituation of orienting to a releasing stimulus may be behaviorally maladaptive in that the animal learns to ignore a biologically important stimulus (e.g., Melzack, 1961). Melzack's solution to this problem was to point out that biologically important stimuli never habituate completely, but, rather, that some residual orienting remains after repeated exposure (Melzack 1961; Russell, 1967; Zeiner \& Peeke, 1969). Melzack has interpreted such habituation as a shift in organization of stimulusrelated behaviors from coarse to fine; the emotional behavior habituates, leaving vigilant behavior intact. In this sense, habituation to a biologically important stimulus shifts the animal to more efficient behavior, and hence is adaptive. This interpretation is supported in two ways by the results reported here: First, orienting to the D100 stimulus did not habituate completely, and second, repeated exposure to the D100 stimulus was accompanied by a progressive shift from freezing to stationary orienting. This shift in behavioral organization from emotional to attentive is just that described by Melzack (1961).

One of the present results-greater recovery of habituation over time to a biologically important stimulus-shows how a second mechanism might operate in preserving an animal's reactivity to biologically important stimuli. Although stimuluslinked attentive behavior may never habituate completely, it is always attenuated markedly with repeated exposure. In the case of exposure to a sufficiently intense biologically important stimulus, however, attentive behavior recovers rapidly over time. This recovery is not to the initial levels, which were characterized by freezing, but to longer durations of orienting. Thus, recovery (at least over periods of up to 7 days) is not based on a return to inefficient behavior dominated by emotional response, but rather is an augmentation of stimulus-related vigilance. 


\section{REFERENCES}

Bolles, R. C. Species-specific defense reactions and avoidance learning. Psychological Review, 1970, 77, 32-48.

Bradley, J. V. Distribution free statistical tests. Englewood Cliffs, N.J: Prentice-Hall, 1968.

Dunham, $\mathbf{P}$. The nature of reinforcing stimuli. In $\mathbf{W}$. $K$. Honig \& J. E. R. Staddon (Eds.), Handbook of operant behavior. Englewood Cliffs, N.J: Prentice-Hall, 1977.

Figle R, M. H. The relation between eliciting stimulus strength and habituation of the threat display in male Siamese fighting fish (Betta splendens). Behaviour, 1972, 42, 63-96.

FiLe, S. E. Inter-stimulus interval and the rate of behavioral habituation. Quarterly Journal of Experimental Psychology, $1973,25,360-367$.

HEBB, D. O. Textbook of psychology. London: Saunders, 1972.

Hinde, R. A. Behavioral habituation. In G. Horn \& R. A. Hinde (Eds.), Short-term changes in neural activity and behavior. Cambridge, England: Cambridge University Press, 1970.

Hinde, R. A., \& Stevenson-Hinde, J. (Eds). Constraints on learning. London: Academic Press, 1973.

Melzack, $R$. On the survival of mallard ducks after "habituation" to the hawk-shaped figure. Behaviour, 1961, 17, 9-16.

Peeke, H. V. S., \& Zeiner, A. R. Habituation to environmental and specific auditory stimuli. Behavioral Biology, 1970, 5, 23-29.
Rozin, P., \& Kalat, J. Specific hungers and poison avoidance as adaptive specializations of learning. Psychological Review, $1971,78,459-486$.

Russelt, E. R. Changes in the behavior of Lebistes reticulatus upon a repeated shadow stimulus. Animal Behaviour, 1967, 15, 574-585.

Seligman, M. E. P. On the generality of the laws of learning. Psychological Review, 1970, 77, 418-486.

Shettleworth, S. J. Constraints on learning. Advances in the Study of Behavior, 1972, 4, 1-68.

Zeinen, A. R., \& PeEke, H. V. S. Habituation of response to distress cries in the rat: Measurement by an innate suppression technique. Communications in Behavioral Biology, 1969, 3, 249-252.

\section{NOTES}

1. Nonparametric methods (Bradley, 1968) were used for statistical analyses, since in all cases the data failed to satisfy the requirement of homogeneity of variance for parametric analysis of variance.

(Manuscript received February 11, 1983; revision accepted for publication June 10, 1983.) 